\title{
Evaluation of Olfactory Bulb Volumes in Patients With Rheumatoid Arthritis: A Retrospective Study
}

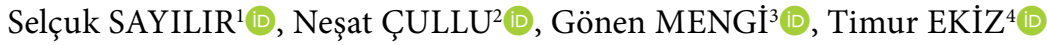 \\ ${ }^{1}$ Department of Physical Medicine and Rehabilitation, Muğla Sıtkı Koçman University Faculty of Medicine, Muğla, Turkey \\ ${ }^{2}$ Department of Radiology, Muğla Sitkı Koçman University Faculty of Medicine, Muğla, Turkey \\ ${ }^{3}$ Department of Rheumatology, Muğla Sıtkı Koçman University Faculty of Medicine, Muğla, Turkey \\ ${ }^{4}$ Department of Physical Medicine and Rehabilitation, Dermancan Medical Center, Adana, Turkey
}

\begin{abstract}
Objectives: This study aims to evaluate olfactory bulb $(\mathrm{OB})$ volume in patients with rheumatoid arthritis (RA) using magnetic resonance imaging. Patients and methods: In this retrospective and case-control study, OB volumes of 37 RA patients ( 6 males, 31 females; mean age $48.6 \pm 10.8$ years; range, 18 to 65 years) were compared with those of 36 healthy control subjects ( 5 males, 31 females; mean age $46.5 \pm 6.9$ years; range, 22 to 62 years). $\mathrm{OB}$ images were gained with a protocol of $256 \times 256$ matrix and a $24-\mathrm{cm}$ field of view, repetition time $=5000 \mathrm{milliseconds}$ (TR $5000 \mathrm{msec}$ ), echo time $=130$ milliseconds (TE $130 \mathrm{msec}$ ), number of excitations=2 (NEX 2) and a $5 \mathrm{~mm}$ slice thickness. OB volume was computed with the aid of the above images using three dimensional views. The surface of each slice area was calculated in $\mathrm{mm}^{2}$ and all surfaces were added and multiplied by front-back length to obtain a volume in $\mathrm{mm}^{3}$.

Results: Left $\left(70.5 \pm 14.4\right.$ vs. $\left.91.1 \pm 12.2 \mathrm{~mm}^{3}\right)$, right $\left(73.9 \pm 15.1\right.$ vs. $\left.91.2 \pm 12.4 \mathrm{~mm}^{3}\right)$, and total $\left(144.5 \pm 27.4\right.$ vs. $\left.182.8 \pm 21.5 \mathrm{~mm}^{3}\right)$ OB volumes were significantly lower in the RA group than in the control group (all $p<0.05$ ).

Conclusion: Patients with RA may be under risk of decreased OB volumes and related impaired odor functions which might affect the quality of life and activities of daily living adversely.

Keywords: Magnetic resonance imaging, olfactory bulb volume, rheumatoid arthritis.
\end{abstract}

Olfactory perception plays an important role to provide environmental communication of the human being. Some specific localizations in the central nervous system are specialized for olfactory functions such as detection, perception and identification of the odor. The olfactory bulb $(\mathrm{OB})$ is the first step of transmission of the olfactory pathway, and it is located bilaterally in the olfactory cortex, hypothalamus, amygdala, and basal telencephalon., ${ }^{1,2}$ The neural progenitor cells have roles of dynamic changes such as neuroplastic changes and synaptogenesis in the $\mathrm{OB}$ in respect of the olfacory functions throughout life as shown in previous animal studies. ${ }^{3}$ Some psychiatric and neurologic disorders, including depression, Alzheimer's disease, schizophrenia, and Parkinson's disease may be related to olfactory dysfunction or decreased volume of the OB. ${ }^{4-6}$ Moreover, decreased $\mathrm{OB}$ volume has been shown in some autoimmune diseases. ${ }^{7-10}$ Magnetic resonance imaging (MRI) studies have demonstrated decreased $\mathrm{OB}$ volumes in patients with olfactory dysfunction as well. ${ }^{11}$ Although impaired olfactory functions such as discrimination, identification, and threshold of the odor have been reported by using self-reported

Received: November 24, 2017 Accepted: February 10, 2019 Published online: April 18, 2019

Correspondence: Selçuk Sayılır, MD. Muğla Sıtkı Koçman Üniversitesi Tıp Fakültesi Fiziksel Tıp ve Rehabilitasyon Anabilim Dalı, 48000 Gülağzı, Muğla, Turkey. Tel: +90 252 - 2141326 e-mail: selcukssay@gmail.com

\section{Citation:}

Sayılır S, Çullu N, Mengi G, Ekiz T. Evaluation of olfactory bulb volumes in patients with rheumatoid arthritis: a retrospective study Arch Rheumatol 2019;34(3):334-337. 
olfactory status with subjective olfactory tests in patients with rheumatoid arthritis (RA) ${ }^{12}$ to our knowledge, $\mathrm{OB}$ volumes have not been studied in RA patients yet. Therefore, in this study, we aimed to evaluate $\mathrm{OB}$ volume in patients with RA using MRI.

\section{PATIENTS AND METHODS}

This retrospective and case-control study was conducted at Muğla Sıtkı Koçman University Training and Research Hospital between May 2013 and May 2017. OB volumes of 37 RA patients (6 males, 31 females; mean age $48.6 \pm 10.8$ years; range, 18 to 65 years) were compared with those of 36 healthy age and sex-matched control subjects ( 5 males, 31 females; mean age $46.5 \pm 6.9$ years; range, 22 to 62 years). Subjects who had any of the following criteria were excluded: brain injury or trauma, depression or other psychiatric conditions, endocrinologic problems, neurological disorders, or chronic sinusitis/rhinitis. Subjects older than 65 years were excluded in respect of age-related degeneration of the OB. ${ }^{13}$ The study protocol was approved by the Muğla Sıtkı Koçman University Clinical Research Ethics Committee (28.09.2017-16/1). Since the data were obtained retrospectively, this study protocol does not require to obtain written informed consent from the participants. The study was conducted in accordance with the principles of the Declaration of Helsinki.

Olfactory bulb volumes were measured using cranial MRI examinations. Total OB volume refers to the sum of the right and left $\mathrm{OB}$ volumes. MRI evaluations were performed with a 1.5-T scanner (GE Healthcare's Signa HDxt 1.5T MRI scanner, Waukesha, Wisconsin, USA). Images were gained with a protocol of $256 \times 256$ matrix and a 24-cm field of view, repetition time $=5000$ milliseconds (TR $5000 \mathrm{msec}$ ), echo time $=130$ milliseconds (TE $130 \mathrm{msec}$ ), number of excitations $=2$ (NEX 2) and a 5-mm slice thickness. $\mathrm{OB}$ volume was computed with the aid of the above images using three dimensional views. The volumetric evaluations were calculated by a 10-year experienced radiologist who was blinded to the subjects. An electronic cursor was used for manually delineating the contours of $\mathrm{OB}$ (Figure 1). The surface of the each slice area was calculated in $\mathrm{mm}^{2}$ and all surfaces were added and multiplied by front-back length to obtain a volume in $\mathrm{mm}^{3}$. Mean of the three consecutive measurements was taken into account. The observer established the minimum of the three consecutive measurements for measuring the MRI images. The intraobserver variability was determined at less than $5 \%$.

\section{Statistical analysis}

Statistical assessments were performed using the IBM SPSS version 20.0 software (IBM Corp., Armonk, NY, USA). Descriptive data were shown as mean \pm standard deviation. Normal distribution was checked with the Shapiro-Wilk test. Independent samples t-test was used for evaluating the statistical differences between the groups and a $p$ value of 0.05 was accepted as statistically significant.

\section{RESULTS}

Left $\left(70.5 \pm 14.4\right.$ vs. $\left.91.1 \pm 12.2 \mathrm{~mm}^{3}\right)$, right $\left(73.9 \pm 15.1\right.$ vs. $\left.91.2 \pm 12.4 \mathrm{~mm}^{3}\right)$, and total $\left(144.5 \pm 27.4\right.$ vs. $\left.182.8 \pm 21.5 \mathrm{~mm}^{3}\right)$ OB volumes were significantly lower in the RA group than in the control group (all $\mathrm{p}<0.05)$ (Table 1).

\begin{tabular}{|c|c|c|c|}
\hline & RA group ( $n=37$ ) & Control group $(n=36)$ & \\
\hline Variable & Mean \pm SD & Mean \pm SD & $p$ \\
\hline Age (year) & $48.6 \pm 10.8$ & $46.5 \pm 6.9$ & 0.319 \\
\hline Right olfactory bulb $\left(\mathrm{mm}^{3}\right)$ & $73.9 \pm 15.1$ & $91.2 \pm 12.4$ & $<0.001$ \\
\hline Left olfactory bulb $\left(\mathrm{mm}^{3}\right)$ & $70.5 \pm 14.4$ & $91.1 \pm 12.2$ & $<0.001$ \\
\hline Total olfactory bulb $\left(\mathrm{mm}^{3}\right)$ & $144.5 \pm 27.4$ & $182.8 \pm 21.5$ & $<0.001$ \\
\hline
\end{tabular}




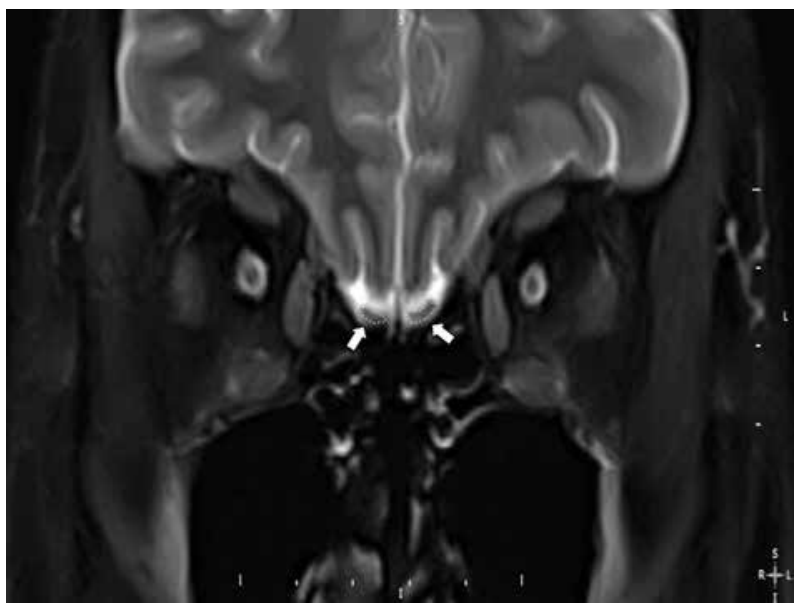

Figure 1. Olfactory bulb measurement with magnetic resonance imaging. Coronal $\mathrm{T}_{2}$-weighted view shows example measuring of olfactory bulb surface area.

\section{DISCUSSION}

In this study, we aimed to explore whether $\mathrm{OB}$ volume of RA patients differ from healthy subjects for the first time in the literature, to the best of our knowledge. The main finding of our study was that RA patients had decreased $\mathrm{OB}$ volumes.

Previous studies showed that impaired olfactory and gustatory functions affect adversely the quality of life. ${ }^{12}$ In addition, decreased $\mathrm{OB}$ volumes are closely associated with impaired olfactory functions (i.e. odor identification, threshold, and discrimination). ${ }^{11} \mathrm{OB}$ can play a role in the stability of the inflammatory responses of the central nervous system. A significant decrease in interleukin (IL)-10 and increase in IL-1b after bulbectomy have been shown in animal models. ${ }^{14}$ From this point of view, an increased inflammatory response of the central nervous system might eventually cause a decrease in $\mathrm{OB}$ volumes in RA.

Systemic corticosteroid utilization may be associated with sodium/water retention and may cause hypokalemic alkalosis which can cause reduced olfactory function. ${ }^{15}$ In addition, chemotherapeutic agents such as cyclophosphamide, methotrexate, and 5-fluorouracil, which are some medical agents for RA treatment, have been associated with impaired olfactory functions. ${ }^{16}$ Therefore, prolongation of the impaired olfactory functions due to utilization of the aforementioned medical treatments may result in decreased $\mathrm{OB}$ volumes in RA patients.

Recently, animal studies have reported that olfactory ecto-mesenchymal stem cells (OMSCs), a new type of resident stem cell in the olfactory lamina propria, can express IL-10 and transforming growth factor-beta. In culture, immunosuppressive effects of OMSCs are associated with suppressing of effector $\mathrm{T}$ cell proliferation and increasing regulatory $\mathrm{T}$ cell development. Transfer of OMSCs reduced arthritis onset and severity, which was accompanied by increased regulatory $\mathrm{T}$ cells and reduced $\mathrm{T}$ helper type 1 (Th1)/Th17 cell responses in vivo. ${ }^{17}$ Therefore, impaired OMSCs functionality may accompany RA which may decrease OMSCs transforming/olfactory functions and related lower OB volumes.

The relationship between reduced $\mathrm{OB}$ volumes and neurodegenerative disorders has been investigated in the literature $e^{7}$ and it has been reported that the effect of the chronic systemic inflammation can play a role in neurodegenerative processes. ${ }^{18}$ Nevertheless, decreased OB volumes in patients with RA can be related to chronic systemic inflammation-associated neurodegenerative processes.

This study has some significant limitations. Our sample size was relatively small and we could not perform analysis for the diagnostic use of $\mathrm{OB}$ volume. Lack of performing odor tests (e.g. Sniffin' Sticks or The University of Pennsylvania Smell Identification Test) is another limitation. Finally, since the data were obtained retrospectively and patients had multiple admissions, we could not obtain sufficient formal and proper data concerning the disease activity, medications, autoantibodies, serologic tests, or systemic involvements.

In conclusion, patients with RA can be under risk of decreased $O B$ volumes and related impaired odor functions which might affect the quality of life and activities of daily living adversely.

\section{Declaration of conflicting interests}

The authors declared no conflicts of interest with respect to the authorship and/or publication of this article. 


\section{Funding}

The authors received no financial support for the research and/or authorship of this article.

\section{REFERENCES}

1. Rolls ET. Taste, olfactory, and food texture processing in the brain, and the control of food intake. Physiol Behav 2005;85:45-56.

2. Martinez-Marcos A. On the organization of olfactory and vomeronasal cortices. Prog Neurobiol 2009;87:2130.

3. Korol DL, Brunjes PC. Unilateral naris closure and vascular development in the rat olfactory bulb. Neuroscience 1992;46:631-41.

4. Doty RL. Olfaction in Parkinson's disease and related disorders. Neurobiol Dis 2012;46:527-52.

5. Moberg PJ, Kamath V, Marchetto DM, Calkins ME, Doty RL, Hahn CG, et al. Meta-analysis of olfactory function in schizophrenia, first-degree family members, and youths at-risk for psychosis. Schizophr Bull 2014;40:50-9.

6. Ortega-Hernandez OD, Kivity S, Shoenfeld Y. Olfaction, psychiatric disorders and autoimmunity: is there a common genetic association? Autoimmunity 2009;42:80-8.

7. Demarquay G, Ryvlin P, Royet JP. Olfaction and neurological diseases: a review of the literature. Rev Neurol (Paris) 2007;163:155-67. [Abstract]

8. Strous RD, Shoenfeld Y. To smell the immune system: olfaction, autoimmunity and brain involvement. Autoimmun Rev 2006;6:54-60.

9. Leon-Sarmiento FE, Bayona EA, Bayona-Prieto J,
Osman A, Doty RL. Profound olfactory dysfunction in myasthenia gravis. PLoS One 2012;7:e45544.

10. Shoenfeld $Y$. To smell autoimmunity: anti-P-ribosomal autoantibodies, depression, and the olfactory system. J Autoimmun 2007;28:165-9.

11. Rombaux P, Duprez T, Hummel T. Olfactory bulb volume in the clinical assessment of olfactory dysfunction. Rhinology 2009;47:3-9.

12. Steinbach S, Proft F, Schulze-Koops H, Hundt W, Heinrich P, Schulz S, et al. Gustatory and olfactory function in rheumatoid arthritis. Scand J Rheumatol 2011;40:169-77.

13. Buschhüter D, Smitka M, Puschmann S, Gerber JC, Witt M, Abolmaali ND, et al. Correlation between olfactory bulb volume and olfactory function. Neuroimage 2008;42:498-502.

14. Song $C$, Leonard BE. The olfactory bulbectomised rat as a model of depression. Neurosci Biobehav Rev 2005;29:627-47.

15. Stanbury RM, Graham EM. Systemic corticosteroid therapy--side effects and their management. $\mathrm{Br} \mathrm{J}$ Ophthalmol 1998;82:704-8.

16. Steinbach S, Hummel T, Böhner C, Berktold $\mathrm{S}$, Hundt $\mathrm{W}$, Kriner $\mathrm{M}$, et al. Qualitative and quantitative assessment of taste and smell changes in patients undergoing chemotherapy for breast cancer or gynecologic malignancies. J Clin Oncol 2009;27:1899-905.

17. Rui K, Zhang Z, Tian J, Lin X, Wang X, Ma J, et al. Olfactory ecto-mesenchymal stem cells possess immunoregulatory function and suppress autoimmune arthritis. Cell Mol Immunol 2016;13:401-8.

18. Perry VH, Cunningham C, Holmes C. Systemic infections and inflammation affect chronic neurodegeneration. Nat Rev Immunol 2007;7:161-7. 